\title{
On Mannheim partner curves in three dimensional Lie groups
}

Ismail Gök, O. Zeki Okuyucu, Nejat Ekmekci, and Yusuf Yayli 


\title{
ON MANNHEIM PARTNER CURVES IN THREE DIMENSIONAL LIE GROUPS
}

\author{
İSMAIL GÖK, O. ZEKI OKUYUCU, NEJAT EKMEKCI, AND YUSUF YAYLI
}

Received 24 September, 2013

\begin{abstract}
In this paper, we define Mannheim partner curves in a three dimensional Lie group $G$ with a bi-invariant metric. The main result of the paper is given as (Theorem 4): A curve $\alpha: I \subset \mathbb{R} \rightarrow G$ with the Frenet apparatus $\{T, N, B, \kappa, \tau\}$ is a Mannheim partner curve if and only if

$$
\lambda \kappa\left(1+H^{2}\right)=1
$$
\end{abstract}

where $\lambda, \mu$ are constants and $H$ is the harmonic curvature function of the curve $\alpha$.

2010 Mathematics Subject Classification: 53A04; 22E15

Keywords: Mannheim curves, Lie groups

\section{INTRODUCTION}

In the classical diferential geometry of curves, J. Bertrand studied curves in Euclidean 3-space whose principal normals are the principal normals of another curve. In [1] he showed that a necessary and sufficient condition for the existence of such a second curve is that a linear relationship with constant coefficients shall exist between the first and second curvatures of the given original curve. In other word, if we denote first and second curvatures of a given curve by $k_{1}$ and $k_{2}$ respectively, then for $\lambda, \mu$ $\in \mathbb{R}$ we have $\lambda k_{1}+\mu k_{2}=1$. Since the time of Bertrand's paper, pairs of curves of this kind have been called Conjugate Bertrand Curves, or more commonly Bertrand Curves (see [10]).

Another kind of associated curve whose principal normal vector field is the binormal vector field of another curve is called Mannheim curve. Mannheim partner curves was studied by Liu and Wang (see [12]) in Euclidean 3- space and in the Minkowski 3-space. After these papers lots of papers have been published about Mannheim curves in Euclidean 3-space, Minkowski 3-space, dual 3-space and Galilean spaces (see [7,9,15,17, 18]). Matsuda and Yorozu [13] gave a definition of generalized Mannheim curve in Euclidean 4-space. They show some characterizations and examples of generalized Mannheim curves. Ersoy et.al. gave a definition of generalized Mannheim curve in Minkowski 4-space. 
The degenarete semi-Riemannian geometry of Lie group is studied by Çöken and Çiftçi [4]. Moreover, they obtanied a naturally reductive homogeneous semiRiemannian space using the Lie group. Then Çiftçi [3] defined general helices in three dimensional Lie groups with a bi-invariant metric and obtained a generalization of Lancret's theorem and gave a relation between the geodesics of the so-called cylinders and general helices.

Recently, Izumiya and Takeuchi [8] have introduced the concept of slant helix in Euclidean 3 -space. A slant helix in Euclidean space $\mathbb{E}^{3}$ was defined by the property that its principal normal vector field makes a constant angle with a fixed direction. Also, Izumiya and Takeuchi showed that $\alpha$ is a slant helix if and only if the geodesic curvature of spherical image of principal normal indicatrix $(N)$ of a space curve $\alpha$

$$
\sigma_{N}(s)=\left(\frac{\kappa^{2}}{\left(\kappa^{2}+\tau^{2}\right)^{3 / 2}}\left(\frac{\tau}{\kappa}\right)^{\prime}\right)(s)
$$

is a constant function.

Harmonic curvature functions have been defined by Özdamar and Hacısalihoğlu [16]. Recently, many studies have been reported on generalized helices and slant helices using the harmonic curvatures in Euclidean spaces and Minkowski spaces $[2,6,11]$. Then, Okuyucu et al. [14] have defined slant helices in three dimensional Lie groups with a bi-invariant metric and obtained some characterizations using their harmonic curvature functions.

In this paper, first of all, we define Mannheim partner curves in a three dimensional Lie group $G$ with a bi-invariant metric and we obtain the necessary and sufficient conditions for the Mannheim partner curves in a three dimensional Lie group $G$.

\section{PReliminaries}

Let $G$ be a Lie group with a bi-invariant metric $\langle$,$\rangle and D$ be the Levi-Civita connection of Lie group $G$. If $\mathfrak{g}$ denotes the Lie algebra of $G$ then we know that $\mathfrak{g}$ is issomorphic to $T_{e} G$ where $e$ is neutral element of $G$. If $\langle$,$\rangle is a bi-invariant metric$ on $G$ then we have

$$
\langle X,[Y, Z]\rangle=\langle[X, Y], Z\rangle
$$

and

$$
D_{X} Y=\frac{1}{2}[X, Y]
$$

for all $X, Y$ and $Z \in \mathfrak{g}$.

Let $\alpha: I \subset \mathbb{R} \rightarrow G$ be an arc-lenghted regular curve and $\left\{X_{1}, X_{2,}, X_{n}\right\}$ be an orthonormal basis of $\mathfrak{g}$. In this case, we write that any two vector fields $W$ and $Z$ along the curve $\alpha$ as $W=\sum_{i=1}^{n} w_{i} X_{i}$ and $Z=\sum_{i=1}^{n} z_{i} X_{i}$ where $w_{i}: I \rightarrow \mathbb{R}$ and $z_{i}: I \rightarrow \mathbb{R}$ are smooth functions. Also the Lie bracket of two vector fields $W$ and $Z$ 
is given by

$$
[W, Z]=\sum_{i=1}^{n} w_{i} z_{i}\left[X_{i}, X_{j}\right]
$$

and the covariant derivative of $W$ along the curve $\alpha$ with the notation $D_{\alpha^{\prime}} W$ is given as follows

$$
D_{\alpha^{\prime}} W=\dot{W}+\frac{1}{2}[T, W]
$$

where $T=\alpha^{\prime}$ and $\dot{W}=\sum_{i=1}^{n} \dot{w}_{i} X_{i}$ or $\dot{W}=\sum_{i=1}^{n} \frac{d w}{d t} X_{i}$. Note that if $W$ is the left-invariant vector field along to the curve $\alpha$ then $W=0$ (see for details [5]).

Let $G$ be a three dimensional Lie group and $(T, N, B, \kappa, \tau)$ denote the Frenet apparatus of the curve $\alpha$. Then the Serret-Frenet formulas of the curve $\alpha$ satisfies:

$$
D_{T} T=\kappa N, \quad D_{T} N=-\kappa T+\tau B, \quad D_{T} B=-\tau N
$$

where $D$ is Levi-Civita connection of Lie group $G$ and $\kappa=\|T\|$.

Definition 1. Let $\alpha: I \subset \mathbb{R} \rightarrow G$ be a parametrized curve. Then $\alpha$ is called a general helix if it makes a constant angle with a left-invariant vector field $X$. That is,

$$
\langle T(s), X\rangle=\cos \theta \text { for all } s \in I,
$$

for the left-invariant vector field $X \in g$ is unit length and $\theta$ is a constant angle between $X$ and $T$, which is the tangent vector field of the curve $\alpha$ (see [3]).

Definition 2. Let $\alpha: I \subset \mathbb{R} \rightarrow G$ be a parametrized curve with the Frenet apparatus $(T, N, B, \kappa, \tau)$ then

$$
\tau_{G}=\frac{1}{2}\langle[T, N], B\rangle
$$

or

(see [3]).

$$
\tau_{G}=\frac{1}{2 \kappa^{2} \tau}\langle\ddot{T},[T, \dot{T}]\rangle+\frac{1}{4 \kappa^{2} \tau}\|[T, \dot{T}]\|^{2}
$$

Proposition 1. Let $\alpha: I \subset \mathbb{R} \rightarrow G$ be an arc length parametrized curve with the Frenet apparatus $\{T, N, B\}$. Then the following equalities

$$
\begin{aligned}
& {[T, N]=\langle[T, N], B\rangle B=2 \tau_{G} B} \\
& {[T, B]=\langle[T, B], N\rangle N=-2 \tau_{G} N}
\end{aligned}
$$

hold [14].

Definition 3. Let $\alpha: I \subset \mathbb{R} \rightarrow G$ be an arc length parametrized curve. Then $\alpha$ is called a slant helix if its principal normal vector field makes a constant angle with a left-invariant vector field $X$ which is unit length. That is,

$$
\langle N(s), X\rangle=\cos \theta \text { for all } s \in I,
$$


where $\theta \neq \frac{\pi}{2}$ is a constant angle between $X$ and $N$ which is the principal normal vector field of the curve $\alpha$ (see [14]).

Definition 4. Let $\alpha: I \subset \mathbb{R} \rightarrow G$ be an arc length parametrized curve with the Frenet apparatus $\{T, N, B, \kappa, \tau\}$. Then the harmonic curvature function of the curve $\alpha$ is defined by

$$
H=\frac{\tau-\tau_{G}}{\kappa}
$$

where $\tau_{G}=\frac{1}{2}\langle[T, N], B\rangle$ (see [14]).

Theorem 1. Let $\alpha: I \subset \mathbb{R} \rightarrow G$ be a parametrized curve with the Frenet apparatus $(T, N, B, \kappa, \tau)$. The curve $\alpha$ is a general helix, if and only if,

$$
\tau=c \kappa+\tau_{G}
$$

where $c$ is a constant (see [3]) or using the definition of the harmonic curvature function of the curve $\alpha$ (see [14]) $H$ is a constant function.

Theorem 2. Let $\alpha: I \subset \mathbb{R} \rightarrow G$ be a unit speed curve with the Frenet apparatus $(T, N, B, \kappa, \tau)$. Then $\alpha$ is a slant helix if and only if

$$
\sigma_{N}=\frac{\kappa\left(1+H^{2}\right)^{\frac{3}{2}}}{H^{\prime}}=\tan \theta
$$

is a constant where $H$ is a harmonic curvature function of the curve $\alpha$ and $\theta \neq \frac{\pi}{2}$ is a constant (see [14]).

\section{MANNHEIM PARTNER CURVES IN A THREE DIMENSIONAL LIE GROUPS}

In this section, we define Mannheim partner curves and their characterizations are given in a three dimensional Lie group $G$ with a bi-invariant metric $\langle$,$\rangle . Also we$ give some characterizations of Mannheim partner curves using the special cases of $G$.

Definition 5. A curve $\alpha$ in 3-dimensional Lie group $G$ is a Mannheim curve if there exists a special curve $\beta$ in 3-dimensional Lie group $G$ such that principal normal vector field of $\alpha$ is linearly dependent binormal vector field of $\beta$ at corresponding point under $\psi$ which is bijection from $\alpha$ to $\beta$. In this case $\beta$ is called the Mannheim partner curve of $\alpha$ and $(\alpha, \beta)$ is called Mannheim curve couple.

The curve $\alpha: I \subset \mathbb{R} \rightarrow G$ in 3-dimensional Lie group $G$ is parametrized by the arc-length parameter $s$ and from the Definition 5 Mannheim mate curve of $\alpha$ is given $\beta: \bar{I} \subset \mathbb{R} \rightarrow G$ in 3-dimensional Lie group $G$ with the help of Figure 1 such that

$$
\beta(s)=\alpha(s)+\lambda(s) N(s), s \in I
$$

where $\lambda$ is a smooth function on $I$ and $N$ is the principal normal vector field of $\alpha$. 


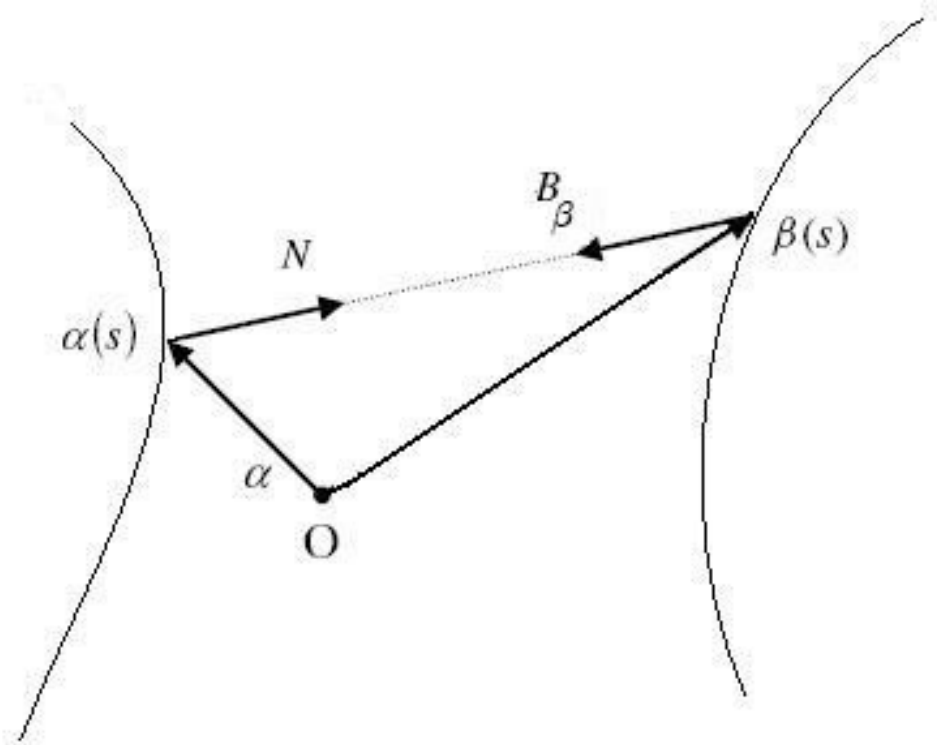

FiguRE 1. Mannheim Partner Curves

Theorem 3. Let $\alpha: I \subset \mathbb{R} \rightarrow G$ and $\beta: \bar{I} \subset \mathbb{R} \rightarrow G$ be a Mannheim curve couple with arc-length parameter $s$ and $\bar{s}$, respectively. Then corresponding points are a fixed distance apart for all $s \in I$, that is,

$$
d(\alpha(s), \beta(s))=\text { constant, for all } s \in I .
$$

Proof. From Definition 5, we can simply write

$$
\beta(s)=\alpha(s)+\lambda(s) N(s)
$$

Differentiating Eq. (3.2) with respect to $s$ and using Eq. (2.3), we get

$$
\begin{aligned}
\frac{d \beta(\bar{s})}{d \bar{s}} \frac{d \bar{s}}{d s} & =\frac{d \alpha(s)}{d s}+\lambda^{\prime}(s) N(s)+\lambda(s) \dot{N}(s) \\
& =T(s)+\lambda^{\prime}(s) N(s)+\lambda(s)\left[D_{T} N-\frac{1}{2}[T, N]\right]
\end{aligned}
$$

and with the help of Proposition 1 and Frenet equations, we obtain

$$
\frac{d \beta(\bar{s})}{d \bar{s}} \frac{d \bar{s}}{d s}=(1-\lambda(s) \kappa(s)) T(s)+\lambda^{\prime}(s) N(s)+\lambda(s)\left(\left(\tau-\tau_{G}\right)(s)\right) B(s)
$$

hence

$$
T_{\beta}(\bar{s})=\frac{d s}{d \bar{s}}\left[(1-\lambda(s) \kappa(s)) T(s)+\lambda^{\prime}(s) N(s)+\lambda(s)\left(\tau-\tau_{G}\right)(s) B(s)\right] .
$$


And then, we know that $\left\{N(s), B_{\beta}(\bar{s})\right\}$ is a linearly dependent set, so we have

$$
\left\langle T_{\beta}(\bar{s}), B_{\beta}(\bar{s})\right\rangle=\frac{d s}{d \bar{s}}\left[\begin{array}{c}
(1-\lambda(s) \kappa(s))\left\langle T(s), B_{\beta}(\bar{s})\right\rangle+\lambda^{\prime}(s)\left\langle N(s), B_{\beta}(\bar{s})\right\rangle \\
+\lambda(s)\left(\tau-\tau_{G}\right)(s)\left\langle B(s), B_{\beta}((\bar{s}))\right\rangle
\end{array}\right]
$$

hence

$$
\lambda^{\prime}(s)=0
$$

that is, $\lambda(s)$ is constant function on $I$. This completes the proof.

Theorem 4. Let $\alpha: I \subset \mathbb{R} \rightarrow G$ be a parametrized curve with arc length parameter $s$ and the Frenet apparatus $(T, N, B, \kappa, \tau)$. Then, $\alpha$ is Mannheim curve if and only if

$$
\lambda \kappa\left(1+H^{2}\right)=1, \text { for all } s \in I
$$

where $\lambda$ is constant and $H$ is the harmonic curvature function of the curve $\alpha$.

Proof. Let $\alpha: I \subset \mathbb{R} \rightarrow G$ be a parametrized Mannheim curve with arc length parameter $s$ then we can write

$$
\beta(s)=\alpha(s)+\lambda N(s)
$$

Differentiating the above equality with respect to $s$ and by using the Frenet equations, we get

$$
\frac{d \beta(s)}{d s}=(1-\lambda \kappa(s)) T(s)+\lambda\left(\tau-\tau_{G}\right)(s) B(s) .
$$

On the other hand, we have

$$
T_{\beta}(\bar{s})=\frac{d \beta}{d s} \frac{d s}{d \bar{s}}=\left[(1-\lambda \kappa(s)) T(s)+\lambda\left(\tau-\tau_{G}\right)(s) B(s)\right] \frac{d s}{d \bar{s}} .
$$

By taking the derivative of this equation with respect to $\bar{s}$ and using the Frenet equations we obtain

$$
\begin{aligned}
\frac{d T_{\beta}}{d \bar{s}}= & {\left[-\lambda \frac{d \kappa}{d s} T(s)+\left(\kappa-\lambda \kappa^{2}-\lambda\left(\tau-\tau_{G}\right)^{2}\right) N(s)+\lambda\left(\tau-\tau_{G}\right)^{\prime} B(s)\right]\left(\frac{d s}{d \bar{s}}\right)^{2} } \\
& +\left[(1-\lambda \kappa(s)) T(s)+\lambda\left(\tau-\tau_{G}\right)(s) B(s)\right] \frac{d^{2} s}{d \bar{s}^{2}}
\end{aligned}
$$

From this equation we get

$$
\begin{gathered}
\left(\kappa-\lambda \kappa^{2}-\lambda\left(\tau-\tau_{G}\right)^{2}\right)=0, \\
\lambda \kappa\left(1+H^{2}\right)=1 .
\end{gathered}
$$

Conversely, if $\lambda \kappa\left(1+H^{2}\right)=1$ then we can easily see that $\alpha$ is a Mannheim curve.

This completes the proof. 
Corollary 1. If $G$ is Abelian Lie group then $\tau_{G}=0$. And so, if $\alpha: I \subset \mathbb{R} \rightarrow G$ is a parametrized Mannheim curve with arc length parameter $s$ and the Frenet apparatus $(T, N, B, \kappa, \tau)$ in Abelian Lie group $G$. Then, $\alpha$ satisfy the following equality

$$
\lambda\left(\kappa^{2}+\tau^{2}\right)=\kappa
$$

Proof. If $G$ is Abelian Lie group then using the $\tau_{G}=0$ and Theorem 4 we have the result.

So, the above Corollary shows that the study is a generalization of Mannheim curves defined by Liu and Wang [12] in Euclidean 3-space.

Theorem 5. Let $\alpha: I \subset \mathbb{R} \rightarrow G$ be a parametrized curve with arc length parameter $s$. Then $\beta$ is the Mannheim partner curve of $\alpha$ if and only if the curvature $\kappa_{\beta}$ and the torsion $\tau_{\beta}$ of $\beta$ satisfy the following equation

$$
\frac{d \kappa_{\beta} H_{\beta}}{d \bar{s}}=\frac{\kappa_{\beta}}{\mu}\left(1+\mu^{2} \kappa_{\beta}^{2} H_{\beta}^{2}\right)
$$

where $\mu$ is constant and $H_{\beta}$ is the harmonic curvature function of the curve $\beta$.

Proof. Let $\alpha: I \subset \mathbb{R} \rightarrow G$ be a parametrized Mannheim curve with arc length parameter $s$ then we can write

$$
\alpha(\bar{s})=\beta(\bar{s})+\mu(\bar{s}) B_{\beta}(\bar{s})
$$

for some function $\mu(\bar{s})$. By taking the derivative of this equation with respect to $\bar{s}$ and using the Frenet equations we obtain

$$
T \frac{d s}{d \bar{s}}=T_{\beta}(\bar{s})+\mu^{\prime}(\bar{s}) B_{\beta}(\bar{s})-\mu(\bar{s})\left(\tau_{\beta}-\tau_{G_{\beta}}\right)(\bar{s}) N_{\beta}(\bar{s})
$$

hence

$$
T \frac{d s}{d \bar{s}}=T_{\beta}(\bar{s})+\frac{d \mu(\bar{s})}{d \bar{s}} B_{\beta}(\bar{s})-\mu(\bar{s}) \kappa_{\beta} H_{\beta} N_{\beta}(\bar{s})
$$

where $H_{\beta}$ is the harmonic curvature function of the curve $\beta$. And then, we know that $\left\{N(s), B_{\beta}(\bar{s})\right\}$ is a linearly dependent set, so we have

$$
\frac{d \mu(\bar{s})}{d \bar{s}}=0 .
$$

This means that $\mu(\bar{s})$ is a constant function. Thus we have

$$
T \frac{d s}{d \bar{s}}=T_{\beta}-\mu(\bar{s}) \kappa_{\beta} H_{\beta} N_{\beta}(\bar{s}) .
$$

On the other hand, we have

$$
T=T_{\beta} \cos \theta+N_{\beta} \sin \theta
$$


where $\theta$ is the angle between $T$ and $T_{\beta}$ at the corresponding points of the curves $\alpha$ and $\beta$. By taking the derivative of this equation with respect to $\bar{s}$ and using the Frenet equations we obtain

$$
\kappa N \frac{d s}{d \bar{s}}=-\left(\kappa_{\beta}+\frac{d \theta}{d \bar{s}}\right) \sin \theta T_{\beta}+\left(\kappa_{\beta}+\frac{d \theta}{d \bar{s}}\right) \cos \theta N_{\beta}+\kappa_{\beta} H_{\beta} \sin \theta B_{\beta} .
$$

From this equation and since $\left\{N(s), B_{\beta}(\bar{s})\right\}$ is a linearly dependent set, we get

$$
\left\{\begin{array}{l}
\left(\kappa_{\beta}+\frac{d \theta}{d \bar{s}}\right) \sin \theta=0 \\
\left(\kappa_{\beta}+\frac{d \theta}{d \bar{s}}\right) \cos \theta=0
\end{array}\right.
$$

For this reason we have

$$
\frac{d \theta}{d \bar{s}}=-\kappa_{\beta} .
$$

From Eq. (3.5), Eq. (3.6) and notice that $T_{\beta}$ is orthogonal to $B_{\beta}$, we find that

$$
\frac{d s}{d \bar{s}}=\frac{1}{\cos \theta}=-\frac{\mu \kappa_{\beta} H_{\beta}}{\sin \theta} \text {. }
$$

Then we have

$$
\mu \kappa_{\beta} H_{\beta}=-\tan \theta \text {. }
$$

By taking the derivative of this equation and applying Eq. (3.7), we get

$$
\mu \frac{d \kappa_{\beta} H_{\beta}}{d \bar{s}}=\kappa_{\beta}\left(1+\mu^{2} \kappa_{\beta}^{2} H_{\beta}^{2}\right)
$$

that is

$$
\frac{d \kappa_{\beta} H_{\beta}}{d \bar{s}}=\frac{\kappa_{\beta}}{\mu}\left(1+\mu^{2} \kappa_{\beta}^{2} H_{\beta}^{2}\right) .
$$

Conversely, if the curvature $\kappa_{\beta}$ and torsion $\tau_{\beta}$ of the curve $\beta$ in three dimensional Lie group $G$ satisfy

$$
\frac{d \kappa_{\beta} H_{\beta}}{d \bar{s}}=\frac{\kappa_{\beta}}{\mu}\left(1+\mu^{2} \kappa_{\beta}^{2} H_{\beta}^{2}\right)
$$

for constant $\mu(\bar{s})$, then we define a curve by

$$
\alpha(\bar{s})=\beta(\bar{s})+\mu B_{\beta}(\bar{s})
$$

and we will show that $\{\alpha, \beta\}$ is Mannheim curve couple in three dimensional Lie group $G$. By taking the derivative of Eq. (3.8) with respect to $\bar{s}$ twice, we get

$$
\begin{gathered}
T \frac{d s}{d \bar{s}}=T_{\beta}-\mu \kappa_{\beta} H_{\beta} N_{\beta}, \\
\kappa N\left(\frac{d s}{d \bar{s}}\right)^{2}+T \frac{d^{2} s}{d \bar{s}^{2}}=\mu \kappa_{\beta}^{2} H_{\beta} T_{\beta}+\left(\kappa_{\beta}-\mu \frac{d \kappa_{\beta} H_{\beta}}{d \bar{s}}\right) N_{\beta}-\mu \kappa_{\beta}^{2} H_{\beta}^{2} B_{\beta},
\end{gathered}
$$


respectively. Taking the cross product of Eq. (3.9) with Eq. (3.10) and noticing that

$$
\kappa_{\beta}-\mu \frac{d \kappa_{\beta} H_{\beta}}{d \bar{s}}+\mu^{2} \kappa_{\beta}^{3} H_{\beta}^{2}
$$

we have

$$
\kappa B\left(\frac{d s}{d \bar{s}}\right)^{3}=\mu^{2} \kappa_{\beta}^{3} H_{\beta}^{3} T_{\beta}+\mu \kappa_{\beta}^{2} H_{\beta}^{2} N_{\beta} .
$$

By taking the cross product of Eq. (3.9) with Eq. (3.11), we get

$$
\kappa N\left(\frac{d s}{d \bar{s}}\right)^{4}=-\mu \kappa_{\beta}^{2} H_{\beta}^{2}\left(1+\mu^{2} \kappa_{\beta}^{2} H_{\beta}^{2}\right) B_{\beta} .
$$

This means that the principal normal vector field of the curve $\alpha$ and binormal vector field of the curve $\beta$ are linearly dependent set. And so $\alpha$ is a Mannheim curve and $\beta$ is Mannheim partner curve of the curve $\alpha$ in three dimensional Lie group $G$.

Proposition 2. Let $\alpha: I \subset \mathbb{R} \rightarrow G$ be an arc-lenghted Mannheim curve with the Frenet vector fields $\{T, N, B\}$ and $\beta: \bar{I} \subset \mathbb{R} \rightarrow G$ be a Mannheim mate of $\alpha$ with the Frenet vector fields $\left\{T_{\beta}, N_{\beta}, B \beta\right.$. Then $\tau_{G}=\tau_{G_{\beta}}$ for the curves $\alpha$ and $\beta$ where $\tau_{G}=\frac{1}{2}\langle[T, N], B\rangle$ and $\tau_{G_{\beta}}=\frac{1}{2}\left\langle\left[T_{\beta}, N_{\beta}\right], B_{\beta}\right\rangle$.

Proof. Let $(\alpha, \beta)$ be a Mannheim curve couple. From Eq. (3.3) we have

$$
T_{\beta}(\bar{s})=\left[(1-\lambda \kappa(s)) T(s)+\lambda\left(\tau-\tau_{G}\right)(s) B(s)\right] \frac{d s}{d \bar{s}} .
$$

We take the norm of this equation and by using Eq. (3.4), we obtain

$$
\frac{d \bar{s}}{d s}=\lambda \kappa H \sqrt{1+H^{2}}
$$

If we consider with together the last two equations, we get

$$
T_{\beta}(\bar{s})=\frac{H}{\sqrt{1+H^{2}}} T(s)+\frac{1}{\sqrt{1+H^{2}}} B(s) .
$$

Since $(\alpha, \beta)$ is a Mannheim curve couple we know $B_{\beta}(\bar{s})=N(s)$. Then,

$$
\begin{gathered}
N_{\beta}(\bar{s})=B_{\beta}(\bar{s}) \times T_{\beta}(\bar{s}) \\
N_{\beta}(\bar{s})=\frac{1}{\sqrt{1+H^{2}}} T(s)-\frac{H}{\sqrt{1+H^{2}}} B(s) .
\end{gathered}
$$

We know from Definition $2\left\langle\left[T_{\beta}, N_{\beta}\right], B_{\beta}\right\rangle=2 \tau_{G_{\beta}}$ for the curve $\beta$. Then with the help of above equations for $T_{\beta}(\bar{s}), N_{\beta}(\bar{s})$ and $B_{\beta}(\bar{s})$, we obtain

$$
\begin{gathered}
\left\langle\left[\frac{H}{\sqrt{1+H^{2}}} T+\frac{1}{\sqrt{1+H^{2}}} B, \frac{1}{\sqrt{1+H^{2}}} T(s)-\frac{H}{\sqrt{1+H^{2}}} B(s)\right], N\right\rangle=2 \tau_{G_{\beta}}, \\
\frac{H^{2}}{1+H^{2}}\langle[T, N], B\rangle+\frac{1}{1+H^{2}}\langle[T, N], B\rangle=2 \tau_{G_{\beta}} .
\end{gathered}
$$


Using the last equation of Proposition 1, we get

$$
\tau_{G}=\tau_{G_{\beta}} .
$$

This completes the proof.

Theorem 6. Let $\alpha: I \subset \mathbb{R} \rightarrow G$ be a parametrized Mannheim curve with curvature functions $\kappa, \tau$ and $\beta: \bar{I} \subset \mathbb{R} \rightarrow G$ be a Mannheim mate of $\alpha$ with curvatures functions $\kappa_{\beta}, \tau_{\beta}$. Then the relations between these curvature functions are

$$
\begin{aligned}
\kappa_{\beta}(\bar{s}) & =\frac{H^{\prime}(s)}{\lambda \kappa(s) H(s)\left(1+H^{2}(s)\right)^{3 / 2}}, \\
\tau_{\beta}(\bar{s}) & =\frac{1}{\lambda H(s)}+\tau_{G_{\beta}} .
\end{aligned}
$$

Proof. If we differentiate Eq. (3.12) and using the Frenet formulas, we have

$$
\kappa_{\beta} N_{\beta} \lambda \kappa H \sqrt{1+H^{2}}=\frac{H^{\prime}}{\left(1+H^{2}\right)^{3 / 2}}(T-H B) .
$$

If we take the norm of the last equation, we get

$$
\kappa_{\beta}=\frac{H^{\prime}}{\lambda \kappa H\left(1+H^{2}\right)^{3 / 2}} .
$$

Since $\{\alpha, \beta\}$ is a Mannheim curve couple, we know $B_{\beta}=N$. If we differentiate this equation and using the Frenet formulas, we have

$$
-\left(\tau_{\beta}-\tau_{G_{\beta}}\right) N_{\beta} \lambda H \sqrt{1+H^{2}}=-T+H B .
$$

If we take the norm of the last equation, we get

This completes the proof.

$$
\tau_{\beta}=\frac{1}{\lambda H}+\tau_{G_{\beta}} .
$$

Theorem 7. Let $\alpha: I \subset \mathbb{R} \rightarrow G$ be an arc-lenghted Mannheim curve and $\beta: \bar{I} \subset$ $\mathbb{R} \rightarrow G$ be a Mannheim mate of $\alpha$. The Mannheim curve $\alpha$ is a slant helix if and only if its Mannheim mate $\beta$ is a general helix.

Proof. If Mannheim curve $\alpha$ is a slant helix, then using Theorem 2 we have, $\sigma_{N}$ is a constant function. From Theorem 6 for the curve $\beta$, we have

$$
\begin{aligned}
\frac{\tau_{\beta}-\tau_{G_{\beta}}}{\kappa_{\beta}} & =\frac{\frac{1}{\lambda H}}{\frac{H^{\prime}}{\lambda \kappa H\left(1+H^{2}\right)^{3 / 2}}} \\
\frac{\tau_{\beta}-\tau_{G_{\beta}}}{\kappa_{\beta}} & =\frac{\kappa\left(1+H^{2}\right)^{3 / 2}}{H^{\prime}}
\end{aligned}
$$




$$
\frac{\tau_{\beta}-\tau_{G_{\beta}}}{\kappa_{\beta}}=\sigma_{N}=\text { constant }
$$

So, the curve $\beta$ which is Mannheim mate of $\alpha$ is a general helix.

Conversely, we assume that $\beta$, which is Mannheim mate of $\alpha$, is a general helix. So we have

$$
\frac{\tau_{\beta}-\tau_{G_{\beta}}}{\kappa_{\beta}}=\text { constant. }
$$

From the last equation and Theorem 6 we can easily see that $\sigma_{N}$ is a constant function. This completes the proof.

Theorem 8. Let $\alpha: I \subset \mathbb{R} \rightarrow G$ be an arc-lenghted Mannheim curve and $\beta: \bar{I} \subset$ $\mathbb{R} \rightarrow G$ be a Mannheim mate of $\alpha$. If $\alpha$ is a slant helix then the harmonic curvature function of the curve $\alpha: H(s)$ is

$$
H(s)=\frac{1}{2}\left(a e^{b s}-\frac{1}{a} e^{-b s}\right)
$$

for some nonzero constant $a$ and $b$ and $s$ is the arc length parameter of the curve $\alpha$. If we consider $a=b=1$, we have the harmonic curvature funtion of the curve $\alpha$ is hyperbolic sine function in arc length $s$, that is, $H(s)=\sinh s$.

Proof. Let $\alpha: I \subset \mathbb{R} \rightarrow G$ be an arc-lenghted Mannheim curve with Frenet apparatus $\{T, N, B, \kappa, \tau\}$ in three dimensional Lie group $G$. Since the curve $\alpha$ is a slant helix, we have

$$
\langle N, X\rangle=\cos \theta, \theta \neq \frac{\pi}{2}
$$

for left invariant vector field $X$. Differentiating Eq. (3.14) twice, we have

$$
-\kappa\langle T, X\rangle+\left(\tau-\tau_{G}\right)\langle B, X\rangle=0
$$

and

$$
-\kappa^{\prime}\langle T, X\rangle+\left(\tau-\tau_{G}\right)^{\prime}\langle B, X\rangle=\left\{\kappa^{2}+\left(\tau-\tau_{G}\right)^{2}\right\}\langle N, X\rangle .
$$

Since $\alpha$ is a Mannheim curve using Theorem 4, we rewrite the last equation

$$
-\kappa^{\prime}\langle T, X\rangle+\left(\tau-\tau_{G}\right)^{\prime}\langle B, X\rangle=\frac{\kappa}{\lambda} \cos \theta
$$

where $\lambda$ is a non-zero constant. By a direct calculation using Eq. (3.15) and Eq. (3.16), we obtain

$$
\langle T, X\rangle=\frac{H}{\lambda H^{\prime}} \cos \theta
$$

and

$$
\langle B, X\rangle=\frac{1}{\lambda H^{\prime}} \cos \theta .
$$

Differentiating Eq. (3.17) and Eq. (3.18), we have

$$
\kappa=\frac{1}{\lambda}\left(1-\frac{H H^{\prime \prime}}{\left(H^{\prime}\right)^{2}}\right),
$$




$$
\tau-\tau_{G}=\frac{H^{\prime \prime}}{\lambda\left(H^{\prime}\right)^{2}},
$$

respectively. These equations give that

$$
H=\frac{\tau-\tau_{G}}{\kappa}=\frac{H^{\prime \prime}}{\left(H^{\prime}\right)^{2}-H H^{\prime \prime}} .
$$

Then we have the following differential equation

$$
\left(1+H^{2}\right) H^{\prime \prime}-\left(H^{\prime}\right)^{2}=0
$$

Solving the last equation, we obtain Eq. (3.13). This completes the proof.

Theorem 9. Let $(\alpha, \beta)$ be a Mannheim curve couple in three dimensional Lie group $G$ with bi-invariant metric. Then $\alpha$ is general helix if and only if $\beta$ is a geodesic.

Proof. If Mannheim curve $\alpha$ is a general helix, then its harmonic curvature $H$ is constant function. And so from Theorem 6 ,

$$
\kappa_{\beta}=0 .
$$

So, the curve $\beta$ is a geodesic.

Conversely we assume that $\beta$ is a geodesic curve. From Theorem 6 we can easily see that

and so

$$
H^{\prime}(s)=0
$$

$$
H(s)=\text { constant }
$$

This completes the proof.

\section{REFERENCES}

[1] M. Bertrand, Mémoire sur la théorie des courbes a double courbure. Comptes Rendus, 1850, vol. 36.

[2] Ç. Camcı, K. İlarslan, L. Kula, and H. H. Hacısalihoğlu, "Harmonic curvatures and generalized helices in $\mathbb{E}^{n}, "$ Chaos Solitons Fractals, vol. 40, no. 5, pp. 2590-2596, 2009.

[3] U. Çiftçi, “A generalization of Lancret's theorem,” J. Geom. Phys., vol. 59, no. 12, pp. 1597-1603, 2009.

[4] A. Çöken and U. Çiftçi, "A note on the geometry of Lie groups," Nonlinear Anal., Theory Methods Appl., Ser. A, Theory Methods, vol. 68, no. 7, pp. 2013-2016, 2008.

[5] P. Crouch and F. Silva Leite, "The dynamic interpolation problem: on Riemannian manifolds, Lie groups, and symmetric spaces," J. Dyn. Control Syst., vol. 1, no. 2, pp. 177-202, 1995.

[6] I. Gök, ${ }_{3} C e t i n$ Camci, and H. Hacisalihoğlu, " $V_{n}$-slant helices in Euclidean $n$-space $E^{n}$." Math. Commun., vol. 14, no. 2, pp. 317-329, 2009.

[7] M. Gungor and M. Tosun, "A study on dual Mannheim partner curves." Int. Math. Forum, vol. 5, no. 45-48, pp. 2319-2330, 2010.

[8] S. Izumiya and N. Takeuchi, "New special curves and developable surfaces," Turk. J. Math., vol. 28, no. 2, pp. 153-163, 2004. 
[9] M. K. Karacan, "Weakened mannheim curves," Int. J. Phys. Sci., vol. 6, no. 20, pp. 4700-4705, 2011.

[10] W. Kühnel, Differentialgeometrie. Kurven - Flächen - Mannigfaltigkeiten, ser. Vieweg Studium: Aufbaukurs Mathematik. Wiesbaden: Vieweg, 1999.

[11] M. Külahc1, M. Bektaş, and M. Ergüt, "On harmonic curvatures of a Frenet curve in Lorentzian space," Chaos Solitons Fractals, vol. 41, no. 4, pp. 1668-1675, 2009.

[12] H. Liu and F. Wang, "Mannheim partner curves in 3-space," J. Geom., vol. 88, no. 1-2, pp. 120 126, 2008.

[13] H. Matsuda and S. Yorozu, "On generalized Mannheim curves in Euclidean 4-space," Nihonkai Math. J., vol. 20, no. 1, pp. 33-56, 2009.

[14] O. Z. Okuyucu, I. Gök, Y. Yaylı, and N. Ekmekci, "Slant helices in three dimensional lie groups," Appl. Math. Comput., vol. 221, pp. 672-683, 2013.

[15] K. Orbay and K. E., "On mannheim partner curves in $e^{3}$," J. Phys. Sci., vol. 4, no. 5, pp. 261-264, 2009.

[16] E. Özdamar and H. Hacisalihoglu, "A charakterization of inclined curves in Euclidean n-space," Commun. Fac. Sci. Univ. Ankara, Ser. A1, Math. Stat., vol. 24, pp. 15-23, 1976.

[17] S. Özkaldi, K. İlarslan, and Y. Yayli, “On Mannheim partner curve in dual space," An. Ştiinţ. Univ. "Ovidius" Constanţa, Ser. Mat., vol. 17, no. 2, pp. 131-142, 2009.

[18] H. B. Öztekin and M. Ergüt, "Null Mannheim curves in the Minkowski 3-space $\mathbb{E}_{1}^{3}$," Turk. J. Math., vol. 35, no. 1, pp. 107-114, 2011.

Authors' addresses

İsmail Gök

Ankara University, Faculty of Sciences, Department of Mathematics, 06100 Ankara, Turkey

E-mail address: igokescience.ankara.edu.tr

O. Zeki Okuyucu

Bilecik Şeyh Edebali University, Faculty of Sciences and Arts, Department of Mathematics, 11210 Bilecik, Turkey

E-mail address: osman. okuyucu@bilecik.edu.tr

Nejat Ekmekci

Ankara University, Faculty of Sciences, Department of Mathematics, 06100 Ankara, Turkey

E-mail address: nekmekciescience.ankara.edu.tr

Yusuf Yaylı

Ankara University, Faculty of Sciences, Department of Mathematics, 06100 Ankara, Turkey

E-mail address: yayliescience.ankara.edu.tr 